\title{
Circuit
}

Musiques contemporaines

Jimmie LeBlanc, Luigi Nono et les chemins de l'écoute : entre espace qui sonne et espace du son. Une analyse de No hay caminos Hay que caminar... Tarkovskij, per 7 cori (1987), Paris-Montréal, L'Harmattan, 2010, 140 p.

\section{Jonathan Goldman}

Volume 21, numéro 1, 2011

Du spirituel dans l'art?

URI : https://id.erudit.org/iderudit/1001167ar

DOI : https://doi.org/10.7202/1001167ar

Aller au sommaire du numéro

Éditeur(s)

Les Presses de l’Université de Montréal

ISSN

1183-1693 (imprimé)

1488-9692 (numérique)

Découvrir la revue

Citer ce compte rendu

Goldman, J. (2011). Compte rendu de [Jimmie LeBlanc, Luigi Nono et les chemins de l'écoute : entre espace qui sonne et espace du son. Une analyse de No hay caminos Hay que caminar... Tarkovskij, per 7 cori (1987), Paris-Montréal, L'Harmattan, 2010, 140 p.] Circuit, 21(1), 89-92. https://doi.org/10.7202/1001167ar d'utilisation que vous pouvez consulter en ligne. 


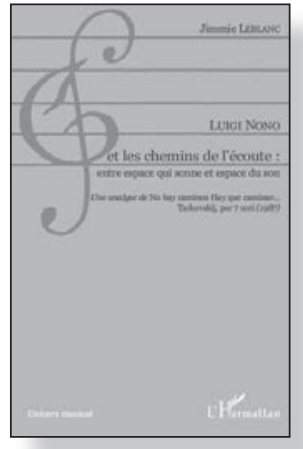

Jimmie LeBlanc

\section{Luigi Nono et les chemins de l'écoute: entre espace qui sonne et espace du son.}

Une analyse de

No hay caminos Hay que caminar...

Tarkovskij, per 7 cori (1987),

Paris-Montréal, L'Harmattan, 2010, 140 p.

Compte rendu de Jonathan Goldman

Malgré le poids de sa réputation, qui le place au même rang que les Boulez, Stockhausen, Maderna, Berio ou Pousseur de ce monde, les œuvres de Luigi Nono (1924-1990) sont rarement jouées à Montréal'. Connu pour ses œuvres gigantesques qui reconfigurent de grandes formes monumentales, telles la cantate et l'opéra, ainsi que pour son engagement communiste, Nono a développé un langage idiosyncrasique intégrant des couches d'allusions inter- et intra-œuvres, à l'instar de György Kurtag, notamment, à travers la pratique d'hommages (comme la référence à Andreï Tarkovski dans le titre de l'œuvre étudiée par l'auteur)². Si Nono compte parmi les géants du temps héroïque du modernisme darmstadtien, il a trouvé, vers la fin de sa vie, une voie hautement personnelle, notamment dans son magnum opus des années 1980, l'œuvre scénique Prometeo, tragedia dell'ascolto (1981-1985)3. Ses œuvres tardives, empreintes d'une lenteur pensive, de nuances à la limite de l'audible, et d'un intérêt grandissant pour la spatialisation et autres effets fournis par l'électronique en temps réel - fruits de ses nombreux passages à l'Experimental Studio de la radio sud-ouest allemand (SWR) à Fribourg-en-Brisgau (Allemagne) - , suscitent un intérêt grandissant auprès des musicologues, des directeurs artistiques et des mélomanes.

Jusqu'à ce jour, la plupart des écrits de et sur Nono ont paru en italien et en allemand4. Applaudissons donc le travail de Jimmie LeBlanc sur l'œuvre tardive No hay caminos Hay que caminar... Tarkovskij (1987) pour orchestre divisé en sept groupes, qui contribue à rendre accessibles l'œuvre et la pensée de Nono à un public francophone. Coup de chapeau également à la maison l'Harmattan pour avoir pris en charge l'édition d'un ouvrage pointu sur la musique, étant donné les difficultés économiques relatives à la publication d'analyses musicales.

L'ouvrage de LeBlanc, de taille relativement modeste, circonscrit en profondeur une seule œuvre du catalogue de Nono, et constitue une belle entrée en matière à l'univers inouï du compositeur italien. Dans cet ouvrage,
1. Grande exception faite du festival "Luigi Nono, maître du son et du silence", sous la direction artistique de Serge Provost, qui a eu lieu au Conservatoire de musique de Montréal du 14 au 20 janvier 2007

2. Sur les réseaux d'allusions et de significations dans les œuvres de Kurtág, voir Friedemann Sallis, "Fleurs recyclées. Sur les traces de relations souterraines dans l'officium breve in memoriam Andreae Szervánszky, opus 28 pour quatuor à cordes de György Kurtág", Circuit, vol. 18, n 1 (2008), p. 45-58. Du même auteur, voir également "Le paradoxe postmoderne et l'œuvre tardive de Luigi Nono", Circuit, vol. 11, $\mathrm{n}^{0} 1$ (2000), p. 69-86.

3. Sur le mythe de Darmstadt, voir le vol. 15, $n^{\circ} 3$ (2005) de Circuit.

4. À l'exception des écrits analytiques et esthétiques de Laurent Feneyrou, qui a également assuré l'édition française des écrits du compositeur: Luigi Nono, Écrits, Laurent Feneyrou (éd. et trad.), Paris, Bourgois, 1993; éd. rév. et augmentée, Genève, Contrechamps, 2007 
5. Stéphane Roy, L'analyse des musiques électroacoustiques; Modèles et propositions, Montréal-Paris, L'Harmattan, 2004

6. Antonio Lai, "Nomos alpha de lannis Xenakis. La matrice disciplinaire et une évaluation contextuelle de l'œuvre", in Présences de Iannis Xenakis, Makis Solomos (dir.), Paris, cdmc, 2001.
7. Sur Kairos 0012512KA. Cet enregistrement est paru en 2007 après la rédaction du mémoire en 2005 , certes, mais tout de même trois années avant la parution du livre. adaptation de son mémoire de maîtrise en composition au Conservatoire de musique de Montréal, le jeune compositeur montréalais, lauréat du prix Jules-Léger de la nouvelle musique de chambre en 2009 , propose un parcours analytique à travers l'œuvre de Nono en se servant d'une grille théorique inspirée à la fois des travaux de Stéphane Roy sur l'analyse de la musique électroacoustiques et de ceux d'Antonio Lai (le concept de «matrice disciplinaire » que LeBlanc a transposé d'une étude que Lai a consacrée à la musique de Xenakis ${ }^{6}$ ). À ces repères théoriques, l'auteur ajoute un autre concept, qu'il baptise «Îlot formel », désignant « un niveau formel supérieur » qui délimite «les grandes sections de l'œuvre tout en s'organisant selon différentes approches de la texture sonore» (p. 15).

En trois chapitres, eux-mêmes divisés en de nombreuses sections et soussections (c'est l'échafaudage d'un mémoire de maîtrise qui apparaît), le livre propose d'abord une analyse synoptique de l'œuvre, dont la pièce de résistance est un long tableau descriptif diachronique étalé sur quinze pages (p. 28-43), qui contient une richesse de détails sur la logique de succession de l'œuvre. Ce tableau accorde une grande attention aux traits immanents de la partition, et montre à quel point l'auteur a bien assimilé, même intériorisé, le langage de Nono.

Ensuite, dans le deuxième chapitre, l'auteur sonde ce qu'il appelle «l'heuristique compositionnelle», cherchant notamment dans les esquisses de l'œuvre, ainsi que dans les commentaires fournis par Nono lui-même, les sources d'inspiration de No hay caminos... Enfin, un bref troisième chapitre propose une synthèse de la pensée musicale de Nono, telle qu'elle s'applique à l'organisation des hauteurs, aux timbres instrumentaux, à la gestion de l'espace et surtout à l'approche du temps musical.

Il suffirait en fait de peu pour que ce livre puisse servir de «guide d'écoute » pour un auditeur curieux muni de rudiments en musique, qui cherche justement des «chemins » d'entrée dans l'œuvre de Nono. Quelques astuces d'ordre pédagogique par-ci, quelques informations contextuelles de plus par-là, auraient pu soulager ce lecteur mélomane qui risque d'être quelque peu dérouté, notamment, par l'absence de référence à l'enregistrement commercial actuellement disponible7. Des informations de base sur l'œuvre, entre autres, la constitution instrumentale exacte de ces « 7 cori» (les groupes instrumentaux) du titre, ne sont guère précisées, même si le lecteur peut les découvrir en consultant les exemples musicaux contenus en annexe du livre. Cela est dommage car LeBlanc a effectué un travail de déblayage, par ailleurs remarquable, de cette partition complexe, en ayant recours à une 
approche sensiblement sémiologique, qui segmente la partition en unités discrètes et décrit leurs profils et leur évolution temporelle.

Lorsque l'auteur s'éloigne d'une description immanente de la partition, pour se diriger vers une perspective plus herméneutique de l'œuvre, certains éléments de mise en contexte manquent ou souffrent d'inexactitudes, comme cette prétendue inspiration chez Nono qui puise dans les «chants judaïques» dont «bon nombre [...] sont en sol (mineur ou majeur)» (p. 48). Or, si la musique de cette tradition orale que constitue le chant de synagogue est parfois effectivement transcrite en sol, ce n'est que pour en faciliter la notation ${ }^{8}$. Par ailleurs, on s'étonne qu'il soit très peu question dans cet ouvrage d'Andreï Tarkovski (dont l'auteur préserve bizarrement l'orthographe italienne). En effet, hormis l'allusion faite au grand cinéaste à même le titre de l'œuvre de Nono et une référence fugitive au «climat d'intériorité et de réflexion d'ordre spirituel » chez Nono qui n'est pas «étranger au climat des œuvres de Tarkovskij» (p. 94), on ne retrouve dans l'ouvrage pratiquement aucune mention du cinéaste. Pourtant, une note de programme de l'œuvre, rédigée par Paolo Petazzi, propose un lien direct entre No hay caminos Hay que caminar... Tarkovskij et le film Le Sacrifice (1986), œuvre ultime du cinéaste russe ${ }^{9}$. Cette absence est regrettable, car l'univers cinématographique aurait pu servir de perche extra-musicale pour aider un lecteur quelque peu intimidé par la complexité de l'écriture de Nono à entrer dans son univers hermétique.

Enfin, regrettons la médiocrité du travail de la maison d’édition, hélas habituelle chez L'Harmattan : l'impression des extraits musicaux est difficilement lisible (les cinq lignes des portées paraissent chacune dans une nuance de gris différente), les normes de références dans les notes et la bibliographie manquent de cohérence, les citations et les exemples musicaux sont souvent trop petits pour être aisément lisibles, etc. Si on félicite L'Harmattan pour le pari pris en faveur des travaux universitaires, on déplore toutefois le manque d'encadrement que cette maison offre à ses auteurs, qui ressemble drôlement à ce que André Schiffrin a appellé «l'édition sans éditeurs ${ }^{10}$ » un phénomène qui se répand de plus en plus aujourd'hui, jusque dans les maisons les plus prestigieuses.

L'ouvrage de LeBlanc se conclut de façon originale sur un recueil de citations de Nono ainsi que d'auteurs de prédilection de celui-ci, tels Edmond Jabès, Massimo Cacciari, Robert Musil, Jean-Paul Sartre, Umberto Eco, ainsi que Tarkovski lui-même. L'auteur dispose les citations tel un long poème continu, tentant par là de s'inspirer des formes fragmentaires si chères à Nono. Si le résultat évoque bien l'univers philosophique que Nono habitait,
8. De même, l'auteur parle d'un $\mathrm{Kol}$ Nidre (prière dont le contour mélodique ressemblerait à ce qu'il nomme le "Neume Microtonal" dans l'œuvre de Nono) qui serait " très fréquemment chantée ou récitée dans toutes les synagogues du monde entier" (p. 48), alors qu'elle n'est chantée qu'à une seule date du calendrier, lors du jour de jeûne Yom Kippur.

9. brahms.ircam.fr/works/ work/10816/\#program

10. André Schiffrin, L'édition sans éditeurs, Paris, La Fabrique, 2001. 
et bien qu'une liste de citations soit fournie en annexe, il rend difficile la tâche d'un lecteur académique qui voudrait faire référence à l'un de ces beaux passages dans un travail ultérieur. Mais cette fin en collage a pour heureux effet de faire de l'ouvrage de LeBlanc une œuvre ouverte en soi et une belle invitation à la découverte de Nono. 